\title{
Support Vector Machines to Accelerate Reflectarray Analysis and Optimization
}

\author{
Daniel R. Prado*†, Jesús A. López-Fernández*, Manuel Arrebola* and Fernando Las-Heras* \\ *Group of Signal Theory and Communications, Universidad de Oviedo, Spain \\ \{drprado, jelofer, arrebola, flasheras\}@ $@$ tsc.uniovi.es \\ ${ }^{\dagger}$ Institute of Sensors, Signals and Systems, Heriot-Watt University, Edinburgh, U.K.
}

\begin{abstract}
The analysis of reflectarray antennas is substantially accelerated by using Support Vector Machines (SVMs) to model the matrix of reflection coefficients in substitution of a fullwave analysis based on local periodicity, the Method of Moments (MoM-LP) in this work. The SVM model takes as input variables two geometrical variables which control the phase-shift for two different polarizations. Thus, the employed model is able to analyze shaped-beam dual-linear polarized reflectarrays. As test case, a shaped-beam reflectarray radiating a pattern for Local Multipoint Distribution Service applications, presenting a squared-cosecant cut in elevation and sectored-beam in azimuth, is presented, showing a high degree of agreement in both the copolar and crosspolar patterns between the simulations of SVM and MoM-LP. Furthermore, the acceleration with regard to the MoM-LP is between three and four orders of magnitude, which demonstrates the suitability of the SVM model approach for reflectarray direct optimization.
\end{abstract}

Index Terms-reflectarray, analysis, synthesis, optimization, Support Vector Machine (SVM), Method of Moments (MoM), local periodicity, shaped-beam pattern

\section{INTRODUCTION}

Advanced applications for reflectarrays require a fast and accurate analysis in order to perform efficient and reliable optimizations [1]. In this regard, the usual Phase-Only Synthesis (POS) is only able to control the shape of the copolar pattern due to the simplifications imposed to obtain a fast optimization [2]. Briefly, the POS only works with the phases of the direct coefficients from the reflection coefficient matrix, assuming no losses and no cross-polarization introduced by the element. They are taken into account at a later step in the design process [1], where no control over the radiation pattern is possible.

In order to account for the crosspolar pattern, the full matrix of reflection coefficients must be considered [3]. This matrix is usually obtained with a full-wave analysis based on local periodicity. In addition, since the crosspolar pattern presents a very low relative value with regard to the copolar pattern, high accuracy in the computation of the reflection coefficient matrix is required, which is the main reason for the use of a full-wave analysis tool. However, even though it has been demonstrated that a reflectarray synthesis using such a tool directly in the optimization loop is feasible within a reasonable time span [3], it would be desirable to accelerate the process. Solutions such as look-up tables [4] or Artificial Neural Networks (ANN) [5] have been proposed to accelerate the analysis of reflectarray unit cells. However, they present some issues. Databases require a very large number of samples

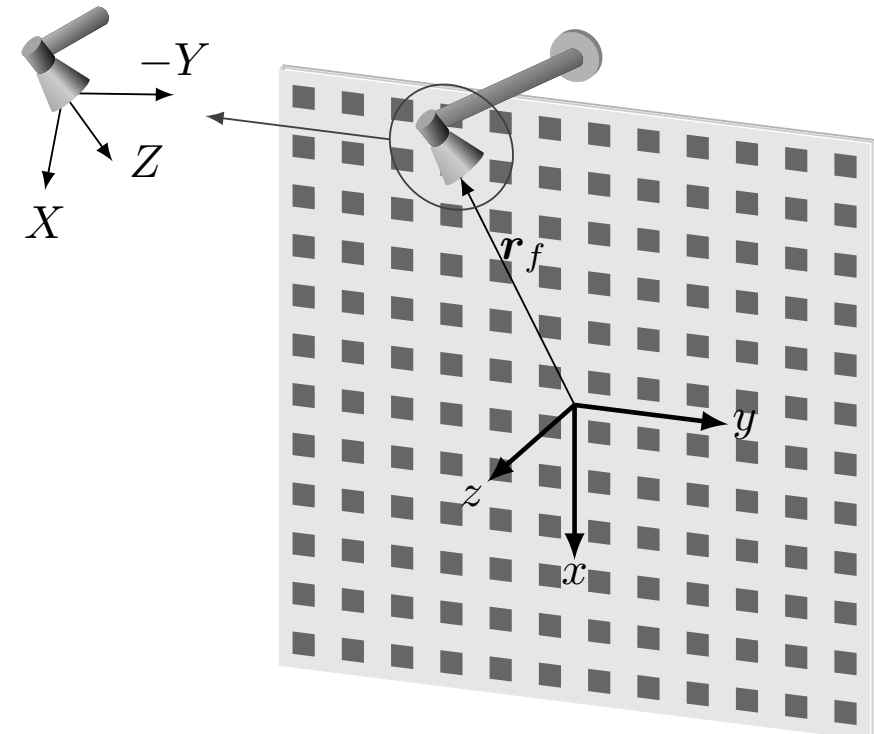

Fig. 1. Representation of a single-offset reflectarray with a horn antenna as feed.

and the use of interpolation produces a loss of accuracy, which is critical in the cross-coefficients of the reflection coefficient matrix. On the other hand, ANNs suffer from overfitting and limited results have been obtained when dealing with the full matrix of reflection coefficients [6].

In this work, we use Support Vector Machines (SVMs) to model a reflectarray unit cell comprised of two sets of four parallel and coplanar dipoles. Each set of dipoles controls the phase-shift for each linear polarization. Thus, the model is able to efficiently and accurately analyze shaped-beam duallinear polarized reflectarrays. Simulations between SVM and MoM-LP show a high degree of agreement in both copolar and crosspolar patterns, which along an obtained acceleration better than three orders of magnitude, demonstrate the usefulness of employing SVMs for the analysis and optimization of reflectarray antennas.

\section{SVM CHARACTERIZATION OF THE UNIT CELL}

\section{A. Reflectarray analysis}

A single-offset reflectarray is considered [1] (see Fig. 1), comprised of a feed (usually a horn antenna) which imposes an incident field $\left(\vec{E}_{\text {inc }}\right)$ on the reflectarray surface, which is 


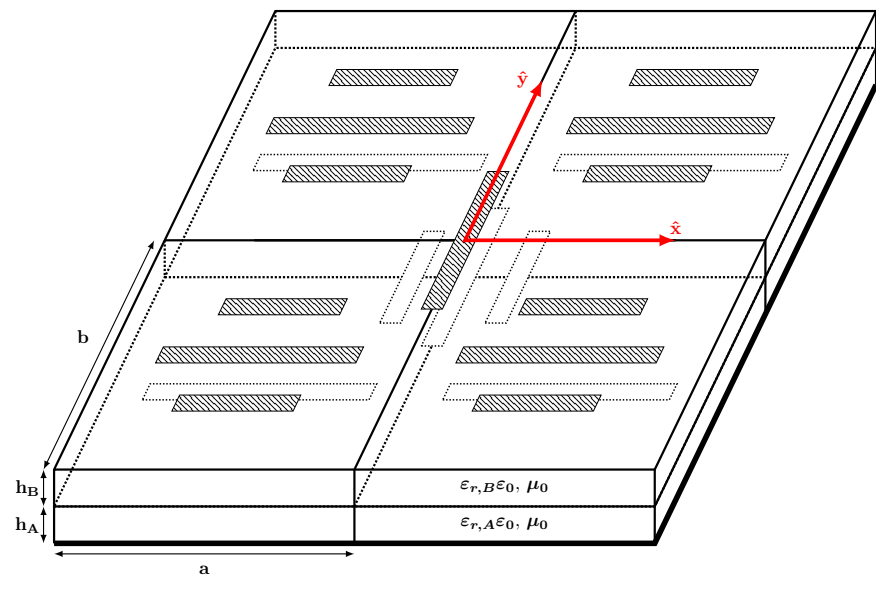

Fig. 2. Employed reflectarray unit cell based on parallel and coplanar dipoles in two layers of metallization.

planar. Thus, the reflected tangential electric field is obtained on each element $(m, n)$ as:

$$
\vec{E}_{\text {ref }}^{X / Y}\left(x_{m}, y_{n}\right)=\mathbf{R}^{m n} \vec{E}_{\text {inc }}^{X / Y}\left(x_{m}, y_{n}\right),
$$

where the superscript indicates the antenna polarization, $\left(x_{m}, y_{n}\right)$ are the coordinates of element $(m, n)$ and

$$
\mathbf{R}^{m n}=\left(\begin{array}{cc}
\rho_{x x}^{m n} & \rho_{x y}^{m n} \\
\rho_{y x}^{m n} & \rho_{y y}^{m n}
\end{array}\right),
$$

is the matrix of reflection coefficient, where $\rho_{x x}^{m n}$ and $\rho_{y y}^{m n}$ are the direct coefficients, and $\rho_{x y}^{m n}$ and $\rho_{y x}^{m n}$ are the crosscoefficients. In this work, we employ the MoM-LP of [7] to obtain samples the $\mathbf{R}^{m n}$ matrix of the unit cell shown in Fig. 2 in order to train the SVM models. The LP assumption is an approximation in which each unit cell is embedded in an infinite array comprised of the same cell and approximately takes into account the losses due to the substrate, mutual coupling between elements, the field reradiated by the element (metallizations) and reflected by the substrate and ground plane.

From the tangential field of (1), the radiation patterns can be efficiently computed using the Fast Fourier Transform [1] and then the copolar and crosspolar components obtained using Ludwig's third definition with the First Principle of Equivalence [3].

\section{B. SVM characterization of the unit cell}

The considered reflectarray unit cell (see Fig. 2) is comprised of two sets of four parallel dipoles each. In order to simplify the SVM model, each set will be controlled by a variable and the dipoles lengths will be proportional to that variable. Thus, we define $T_{x}$ and $T_{y}$ as the variables which control the lengths of the dipoles oriented in the $\hat{x}$ and $\hat{y}$ directions, respectively. This way, the dimensionality of the problem is also reduced, which helps to obtain a more accurate model. In addition, the reflection coefficients vary depending on the angle of incidence of the impinging wave with which the unit cell is analyzed [1]. Instead of considering the incident angle as a variable in the SVM model, a model will be generated for each incident angle. Finally, the chosen substrate is the CuClad 233, which has a thickness of $0.787 \mathrm{~mm}$ and complex relative permittivity of $\epsilon_{r}=2.33-j 3.029 \cdot 10^{-3}$, and is used for both layers. The working frequency is set to $25.5 \mathrm{GHz}$ and the periodicity of the cell is $5.84 \mathrm{~mm}$ in both directions.

The LIBSVM library [8] is employed to generate models of the real and imaginary parts of the four reflection coefficients in (2). Also, in order to further improve the SVM model and due to the smooth behavior shown, the magnitudes of $\rho_{x x}$ and $\rho_{y y}$ are also modeled, having $10 \mathrm{SVM}$ models per angle of incidence. For the SVM training, 1750 randomly generated samples are used per angle of incidence. A novel training strategy is followed which resulted in a mean training time of 70 seconds per SVM.

\section{Antenna Analysis Results}

\section{A. Antenna specifications}

For the radiation pattern simulations, a single-offset rectangular reflectarray is employed, as the one depicted in Fig. 1. The reflectarray is comprised of 900 elements which are arranged in a regular grid of $30 \times 30$ elements. The feed is modeled with a $\cos ^{q} \theta$ function, imposing an illumination taper of $-19.5 \mathrm{~dB}$ at the edge of the reflectarray. In addition, the feed phase center is placed at $\vec{r}_{f}=(-94,0,214) \mathrm{mm}$ (see Fig. 1). Finally, as previously stated, one SVM model is generated per angle of incidence. Thus, in order to limit the number of models, the incident angles are discretized in $\theta$ and $\varphi$ in steps of $10^{\circ}$. This way, there are only $57(\theta, \varphi)$ angle combinations.

\section{B. Radiation patterns}

The goal of modeling the reflectarray unit cell with SVMs is to be able to compute the radiation patterns efficiently and accurately. In this work, the radiation patterns will be computed employing the First Principle of Equivalence [3] for both polarizations. As an example, a shaped pattern for Local Multipoint Distribution Service (LMDS) applications is considered. This radiation pattern presents a squared-cosecant cut in elevation and a sectored-beam pattern in azimuth.

Fig. 3 shows the three dimensional radiation patterns for $\mathrm{X}$ polarization simulated with both MoM-LP and SVM. The radiation patterns were computed in a far field UV grid of $512 \times 512$ points, representing only those belonging to the visible region. The results show how similar the radiation patterns are, including the crosspolar pattern which is more than $25 \mathrm{~dB}$ below the maximum gain. Similar results were obtained for Y polarization.

To better assess the accuracy of the radiation pattern predicted by the SVMs, the relative error between the MoMLP and SVM simulations is calculated with the following expression:

$$
R E=\frac{\left\|E_{\mathrm{MoM}-\mathrm{LP}}-E_{\mathrm{SVM}}\right\|}{\left\|E_{\mathrm{MoM}-\mathrm{LP}}\right\|},
$$




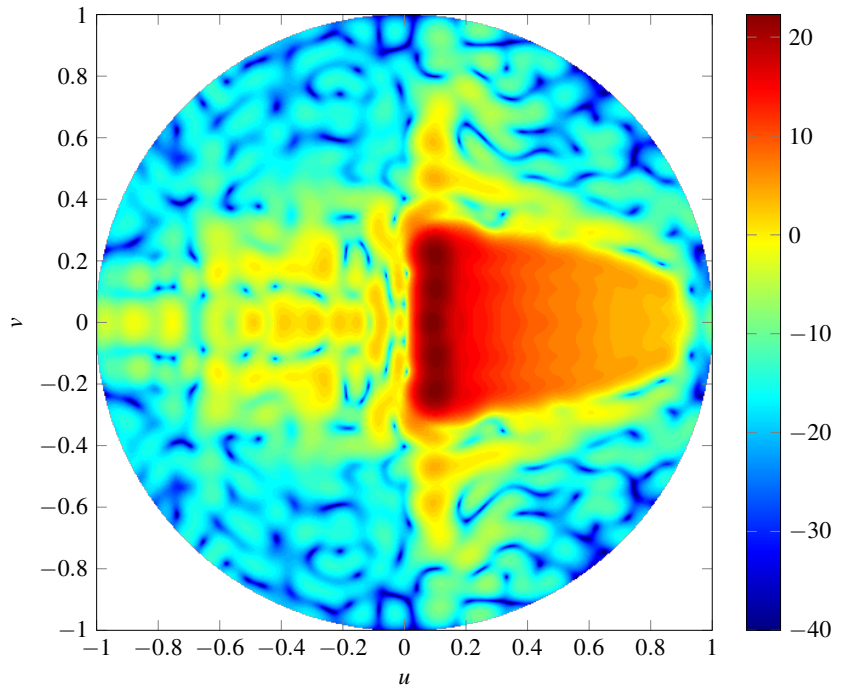

(a)

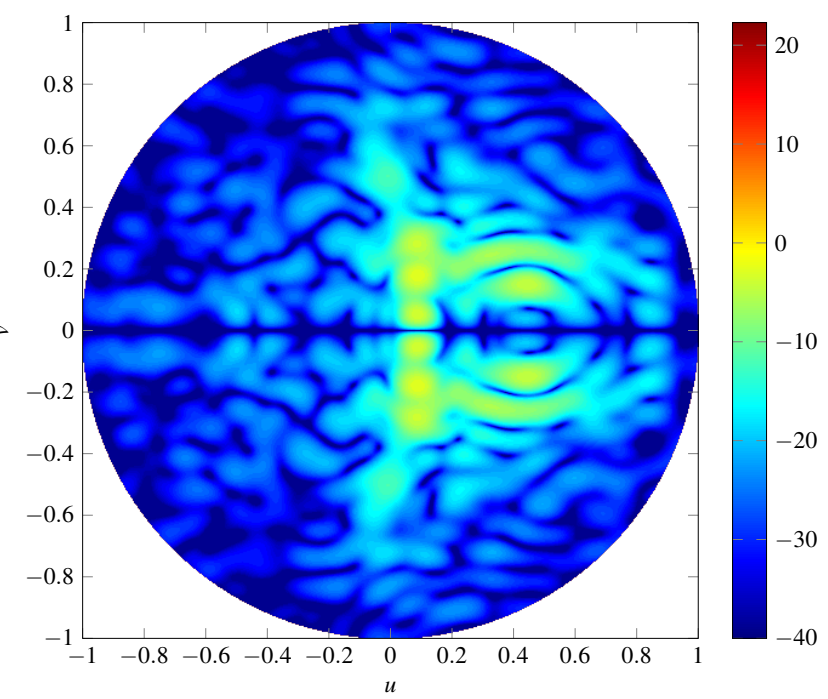

(c)

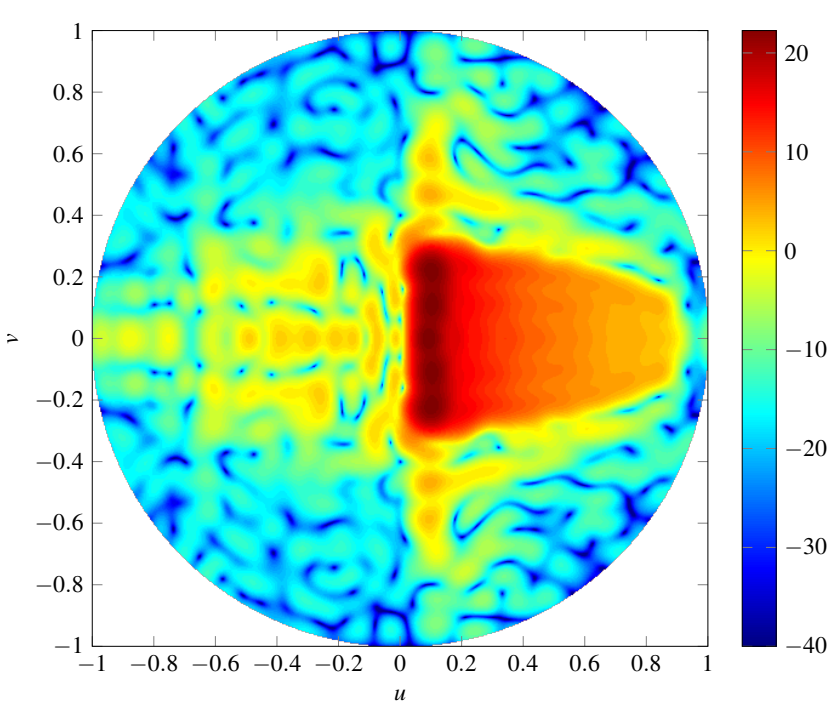

(b)

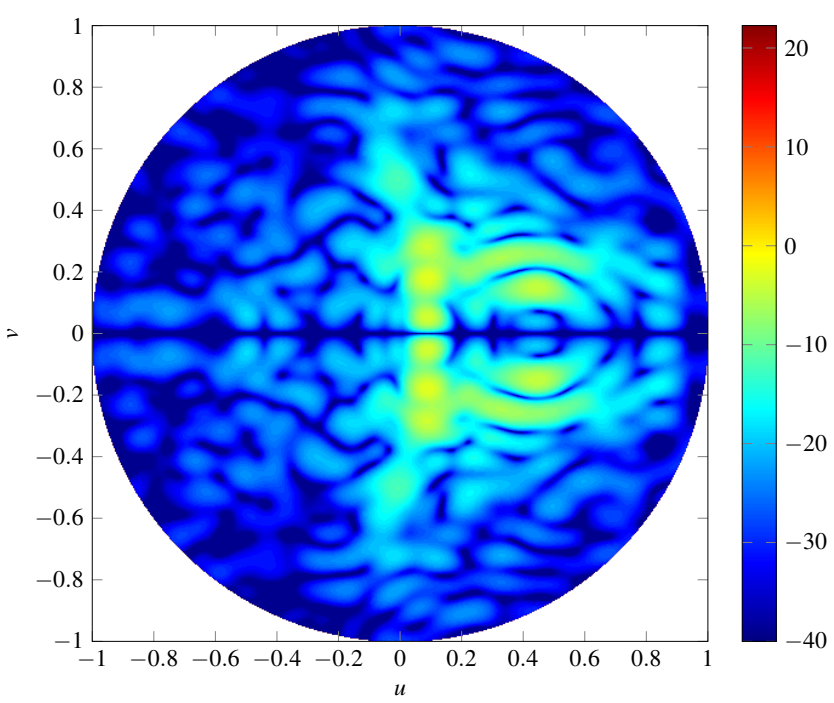

(d)

Fig. 3. Radiation patterns in gain (dBi) for polarization X simuluated with MoM-LP and SVM. (a) Copolar (MoM). (b) Copolar (SVM). (c) Crosspolar (MoM). (d) Crosspolar (SVM). Similar results were obtained for polarization Y.

where $\|\cdot\|$ denotes the Euclidean norm and the radiation pattern computed with MoM-LP is considered the reference since the SVMs were trained using samples of the reflection coefficient matrix simulated with MoM-LP. The relative error was computed for both components of the far field as well as both linear polarizations. For the copolar pattern, an error of $0.50 \%$ was obtained for $\mathrm{X}$ polarization, while it was $0.57 \%$ for $\mathrm{Y}$ polarization. The crosspolar pattern yielded errors of $1.08 \%$ and $1.16 \%$ for $\mathrm{X}$ and $\mathrm{Y}$ polarizations, respectively. Due to the high accuracy of the SVM model, the relative errors of the far field are very low.

Fig. 4 shows the difference in $\mathrm{dB}$ of the LMDS radiation pattern between the SVM and MoM-LP simulations shown in Fig. 3 for polarization X. The difference was computed in linear scale normalized with respect to the MoM-LP simulation and then converted to a logarithmic scale. As it can be seen, the error error is very low in the whole visible region, and specially in the coverage area for the copolar pattern.

Finally, the analysis of the reflectarray using SVMs is sped up by a factor larger than three orders of magnitude. In particular, the analysis was parallelized and carried out in an Intel Core i3-2100 with 4 CPU working at $3.1 \mathrm{GHz}$. The analysis with MoM-LP of the reflectarray comprised of 900 elements took 22.3 seconds, while for the SVMs only took 0.0085 seconds, which supposes a speed-up factor of 2623 . The analysis was also performed without parallelization, and it took 84.5 seconds using MoM-LP and 0.031 seconds using SVMs, having this time a speed-up factor of 2725. Thus, the SVM model is most suitable for performing reflectarray optimization with the goal of reducing the crosspolar pattern 


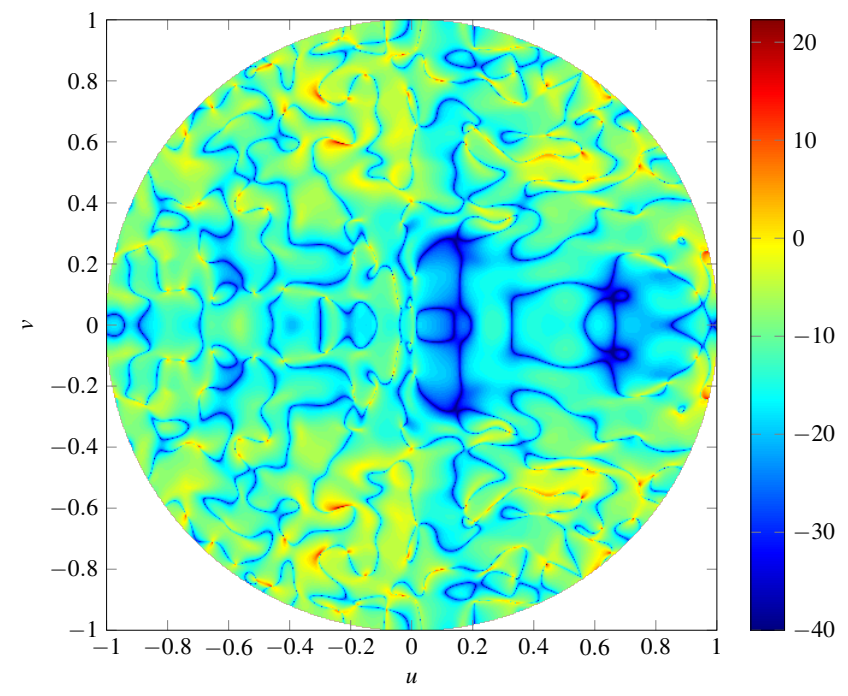

(a)

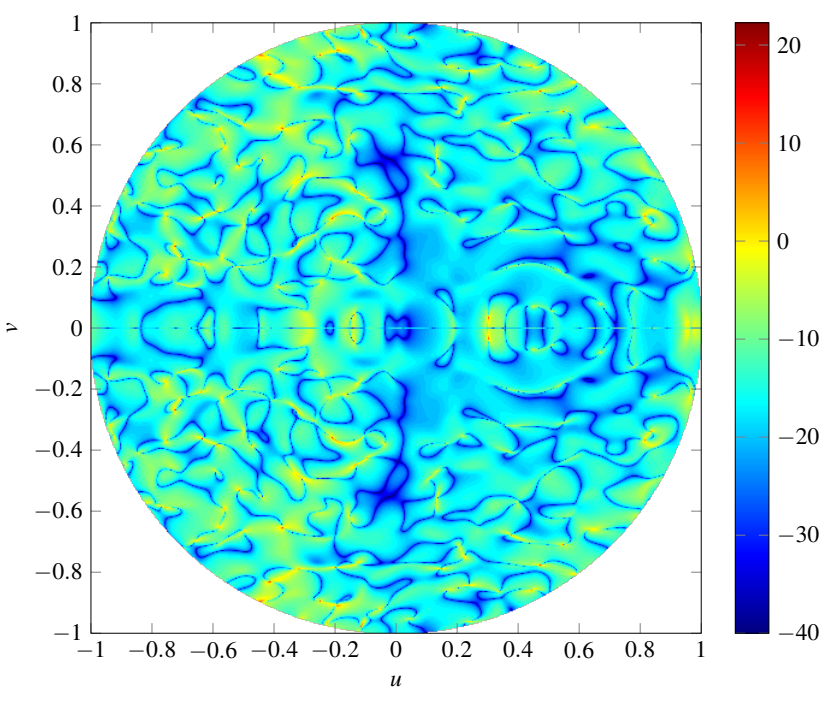

(b)

Fig. 4. Difference in $\mathrm{dB}$ between the SVM and MoM-LP simulations of the LMDS radiation pattern for polarization X. (a) Copolar. (b) Crosspolar.

[3], since it can considerably accelerate the computations while providing high accuracy in the prediction of the radiation patterns, especially the crosspolar component, as shown in Figs. 3 and 4. In this regard, the SVMs would accelerate the computation of the gradient of the optimization algorithms which, for instance, in [3] is the Levenberg-Marquardt (within the framework of the generalized Intersection Approach [9]); and in [4] the gradient minimax.

\section{CONCLUSION}

In this work, Support Vector Machines (SVMs) have been used to efficiently and accurately analyze reflectarray antennas. The SVM model allows to analyze shape-beam dual-linear polarized reflectarray antennas faster than a full-wave Method of Moments based on Local Periodicity (MoM-LP). The speed up factor is better than three orders of magnitude and the simulations show a high degree of agreement between the SVM model and MoM-LP in both, copolar and crosspolar pattern, which demonstrates the suitability of SVMs for the design and optimization of reflectarray antennas.

\section{ACKNOWLEDGMENT}

This work was supported in part by the European Space Agency (ESA) under contract ESTEC/AO/1-7064/12/NL/MH; by the Ministerio de Economía y Competitividad (Spanish Government), under projects TEC2014-54005-P (MIRIIEM) and TEC2016-75103-C2-1-R (MYRADA); by the Gobierno del Principado de Asturias / FEDER under project GRUPIN14114; and by the Gobierno del Principado de Asturias through Programa "Clarín" de Ayudas Postdoctorales / Marie CourieCofund under project ACA17-09.

\section{REFERENCES}

[1] J. Huang and J. A. Encinar, Reflectarray Antennas. Hoboken, NJ, USA: John Wiley \& Sons, 2008.

[2] D. R. Prado, J. Álvarez, M. Arrebola, M. R. Pino, R. G. Ayestarán, and F. Las-Heras, "Efficient, accurate and scalable reflectarray phase-only synthesis based on the Levenberg-Marquardt algorithm," Appl. Comp. Electro. Society Journal, vol. 30, no. 12, pp. 1246-1255, Dec. 2015.

[3] D. R. Prado, M. Arrebola, M. R. Pino, R. Florencio, R. R. Boix, J. A Encinar, and F. Las-Heras, "Efficient crosspolar optimization of shapedbeam dual-polarized reflectarrays using full-wave analysis for the antenna element characterization," IEEE Trans. Antennas Propag., vol. 65, no. 2, pp. 623-635, Feb. 2017.

[4] M. Zhou, S. B. Sørensen, O. S. Kim, E. Jørgensen, P. Meincke, and O. Breinbjerg, "Direct optimization of printed reflectarrays for contoured beam satellite antenna applications," IEEE Trans. Antennas Propag., vol. 61, no. 4, pp. 1995-2004, Apr. 2013.

[5] P. Robustillo, J. Zapata, J. A. Encinar, and J. Rubio, "ANN characterization of multi-layer reflectarray elements for contoured-beam space antennas in the Ku-band," IEEE Trans. Antennas Propag., vol. 60, no. 7, pp. 3205-3214, Jul. 2012.

[6] P. Robustillo, J. Zapata, J. A. Encinar, R. Florencio, R. R. Boix, and J. R. Mosig, "Accurate characterization of multi-resonant reflectarray cells by artificial neural networks," in The $8^{\text {th }}$ European Conference on Antennas and Propagation (EUCAP), The Hague, The Netherlands, Apr. 6-11, 2014, pp. 2297-2299.

[7] R. Florencio, R. R. Boix, and J. A. Encinar, "Enhanced MoM analysis of the scattering by periodic strip gratings in multilayered substrates," IEEE Trans. Antennas Propag., vol. 61, no. 10, pp. 5088-5099, Oct. 2013.

[8] C.-C. Chang and C.-J. Lin, "LIBSVM: A library for support vector machines," ACM Trans. Intell. Syst. Technol., vol. 2, no. 3, pp. 27:1-27:27, Apr. 2011, software available at http://www.csie.ntu.edu.tw/ cjlin/libsvm.

[9] O. M. Bucci, G. D'Elia, G. Mazzarella, and G. Panariello, "Antenna pattern synthesis: a new general approach," Proc. IEEE, vol. 82, no. 3, pp. 358-371, Mar. 1994. 